\title{
Embracing the Electronic Journal: One Library's Plan
}

\author{
Gail McMillan
}

The work of serialists is rarely static and now another exciting new challenge awaits us: the electronic journal. In this paper "electronic journals," also called e-journals, will mean any serials produced, published, and distributed nationally and internationally via electronic networks such as Bitnet and the Internet.' Electronic journals offer many potential benefits, including timely document delivery, direct links from online catalogs, less expensive subscription prices, and easy gathering of statistics on their use. They also pose new problems, such as: How does a library provide access? Does the library's computer have room for complete electronic journals without making sacrifices such as response time or implementation of new software? Who will archive these e-journals and how will this be accomplished?

From the point of view of the medium-sized, academic research library at Virginia Tech, the electronic journal is seen as another technological advancement to be incorporated into the collection of information sources already available from the library. Considering its mission, resources, and users, University Libraries has decided on a "near-term" approach to electronic journal access for the community we serve.

Two years ago the Scholarly Communications Project began at Virginia Tech "to explore, through management of actual journals, new ways of scholarly communication to reduce the expense of distributing print journals through commercial publishers."2 Last

Gail McMillan is Serials/Maintenance Team Leader, University Libraries, P. O. Box 90001, Virginia Polytechnic Institute and State University, Blacksburg, VA 24062-9001. 
summer when University Libraries learned that the Project was going to issue an electronic journal, many of us at the Libraries already knew that we had the resources and expertise to handle this and other electronic publications. We did not question whether we should welcome this new format into our library; rather, our questions were how, when, and where?

To address these questions and others, the University Librarian established the Task Force on the Electronic Journal. In a memo to the task force chair, he charged the group "to investigate and recommend how electronic journals can be integrated into library processes and procedures." 3 He urged us to be creative and act quickly.

The task force consisted of faculty and classified staff from each of the principal areas that would process and handle electronic publications. It was chaired by the Principal Bibliographer and had representatives from the following departments: Reference, Systems Operations, Automation Services, Database Administration, Acquisitions, and Serials Team/Cataloging, which I represented. ${ }^{4}$ From the first task force meeting last October, it was evident that everyone was enthusiastic about the potential of online publications. Within six months the task force had delivered its report ${ }^{5}$ and was invited to begin testing after the Libraries' management committee discussed our recommendations at a public forum. Present at that meeting were all the Libraries' department heads, assistant directors, members of the Task Force on the Electronic Journal and the University's Vice-President for Information Systems. After clarification of some issues, the University Librarian accepted our recommendations and reassigned the task force to oversee implementation. What follows are most of our recommendations, decisions, and accomplishments as of June 13, 1991.

From our initial, free-flowing discussion we quickly agreed on several points. First, an electronic journal should remain online at every step of the way, from internal processing through the point of use by the Libraries' patrons. Therefore, any consideration of the Libraries' printing to paper, binding, or where or how to shelve these publications, was moot. In fact, the task force report referred to printing to paper as "retrograde" and not a business our library should engage in. Second, an electronic journal is a serial and in 
most respects does not need to be treated any differently from serials in hard copy, microform, or CD-ROM formats. A third point was the identification of the appropriate medium for storing the electronic contents of the journals. The task force's discussions for the next several months focused on storage of and access to electronic journals.

In our final report we wrote:

The Task Force on the Electronic Journal believes that, as agents standing between information producers and information consumers, libraries should support electronic journals. The fundamental reason for this is simply that libraries exist as conveyors of academically relevant information. We have not previously discriminated against information because of its format, and this is not the time to start. Furthermore, as customers of information, we want to encourage a means of dissemination which has the potential for considerable cost savings. Finally, we are sensitive to the complexities of bibliographic control and the perishability of unarchived information and we fear that information in electronic form will be at risk of disuse or destruction without the intervention and support of information professionals. ${ }^{5}$

The task force recognized that electronic journals have their limitations. For example, they require hardware and software. Furthermore, electronic transmission of graphics is a problem. Graphics can be mixed with text and transmitted as a Postscript file, but when Postscript has been used for electronic journals, file transfer problems between computer systems have resulted. The electronic journals we initially want to receive do not yet have graphics and are all available in ASCII format. ASCII file transfer between systems has not been a problem. When graphics file transfer protocols become standardized, we will be in a better position to make illustrated electronic journals available to our patrons. In the meantime, transmission of graphics files poses a problem, but graphics per se are not a limitation of the storage options we considered. Ultimately, the information industry will establish patterns and protocols to which libraries will adapt. 
Keeping in mind these and other limitations of electronic journals, we identified several storage and access options and selected four as the most likely to meet our needs.

1. PC-based local area network. Storage and public access could be provided by expanding the existing PC-based Local Area Network (LAN), designed for our CD-ROM network. For over a year our Electronic Reference Area has used the LAN, which provides access to several (currently more than sixteen) CD-ROMs. As many as eight users may simultaneously search these databases from workstations in the main and branch libraries. Soon users outside the library-wide system will also have access to the CD-ROM network. Electronic journals could be copied onto the hard drive of this network server and made available as a selection added to the existing menu; but some development work would be necessary to determine the best method for data management, retrieval on the network, and off-campus access to the network.

The task force considered lags in system response time as the principal disadvantage to this PC-based LAN. As the number of electronic journals increased, additional disk space would be required to store the journals. If demand for access increased, a shared system for both electronic journals and CD-ROMs might become impractical. Response time could gradually increase until users would no longer be able to effectively search either database. A similar but separate network would then be necessary, thus increasing equipment and support costs.

2. PC-based local bulletin board system. Another storage and access option the Task Force on the Electronic Journal considered was a local bulletin board system. We liked this model because it was the least restrictive system, providing university-wide access via the campus network (the CBX), as well as in-house access at a public use workstation. Our systems representatives were confident they could design the bulletin board, without restricting the file format of e-journals (i.e., ASCII, Postscript, etc.). Disadvantages to the bulletin board system, however, include the difficulty of maintaining yet another network within the library, the cost of software, and the possible cost of hardware if no existing microcomputer workstation was available for this project.

3. The Libraries' mainframe computer. The task force consid- 
ered using the Libraries' Hewlett-Packard 300, series 960 mainframe with VTLS, the Libraries' online catalog (an obvious choice for storage as well as access to electronic journals). It would be the optimal situation for both University Libraries and our patrons. With the online catalog as a gateway to the full text of electronic journals, we visualized achieving total integration of bibliographic information and document delivery. It would be ideal for VTLS users, who for years have been able to access our system both locally and remotely through the University's mainframe computer, to access the contents of electronic journals from the online catalog. From the Libraries' perspective, such integration would emphasize our responsibility and role in document delivery, establishing our ability to deliver traditional services in a nontraditional environment. The Libraries' retention of full responsibility would also guarantee that bibliographic and preservation issues receive proper attention.

Storing electronic journals on the Libraries' computer is not now currently a viable option, because most patrons cannot switch easily between the online catalog and source files. New programs would need to be written in order to give VTLS users direct access to the contents of electronic journals, and the task force recommended that the administration pursue this.

An alternate VTLS option would be to copy electronic journals into a separate database, providing VTLS users with an initial menu of options including the online catalog and electronic journals. VTLS users who select the electronic journal option would then see a menu of journal titles. Selecting a title would lead to the table of contents from which to select an article.

However, University Libraries currently has the largest database in the world running on the Hewlett-Packard 3000, series 960. Both of these options employing VTLS as the storage and access medium would require considerable additional resources, such as the purchase of more disk drives at a time when money is very limited and other system priorities such as keyword searching outrank electronic journal access.

4. The University mainframe computer. The task force considered a fourth option for access and storage, the University's IBM 3090-300E mainframe computer with a RoLM CBX, referred to 
here as VM for Virtual Machine. This option has several advantages: (1) Virginia Tech is "heavily wired" and VM is readily available from offices both on and off campus. For example, whether in southwest Virginia at the Blacksburg campus or at the northern Virginia campus, every library staff member has a user ID and is expected to check regularly for electronic mail. Many faculty also use VM for on-campus e-mail as well as national and international communications with their colleagues through Bitnet and the Internet. (2) The means already exist for storing and transmitting large files, whether data, text, or graphics, and many of us regularly transfer, download, and print files. These procedures are routine and already well understood. (3) The electronic journals would be received and posted online with relatively low maintenance (i.e., no uploading or downloading of files from one computer or format to another). (4) Data storage costs would be relatively low since VM computing resources are not presently part of the Libraries' operating budgets. The task force, therefore, concluded that with VM we could readily make electronic journals available.

VM at Virginia Tech also has disadvantages: (1) It is outside the control of the Libraries, ${ }^{6}$ and (2) its editing, word searching and printing capabilities have distinct limitations. (3) At this time access to VM is limited to those faculty, staff, and students who have user IDs. However, anonymous access will soon be available so that the university community can more readily access INFO, the online information system. INFO includes the menu option LIBRARY, which accesses another menu of news and information relating to University Libraries. Electronic journals could also be posted here.

Based on the advantages and disadvantages of the four options considered, the task force recommended that the text of electronic journals be made available on VM, accessible from the University Libraries' information display system, INFO LIBRARY.

The availability of electronic journals through VM would automatically facilitate access by a large component of the university community. But we realized that, in spite of the extensive automation within University Libraries and on our campus, a large component of faculty, not to mention students, are without terminals, mainframe accounts, or both.

Therefore, the task force agreed it was imperative that the Libraries also provide access to patrons lacking user IDs or access to 
terminals. We recommended that the library provide terminals with anonymous access to VM and INFO LIBRARY; access would still be limited, however, because these patrons would be required to come to the library and use the terminals provided. We recommended that the Libraries initially provide two terminals, one in the Electronic Reference Area and one in the Science Reference Area, The necessary wiring and lines are already in place. From these or any other terminals with a VM connection, users could choose the "E-JOURNL" selection from the INFO LIBRARY directory and get a list of the electronic journals available. Selecting a journal title would lead to a list of article titles that could be read at the terminal, downloaded to diskettes, or printed.

Although we acknowledged that the Reference Department would want to weigh this service in the context of other services they provide in the electronic environment, the task force anticipated that direct costs to download onto diskette or to print to paper would be the patron's responsibility. Rather than putting printers at these terminals, printing could be centralized; perhaps the Photocopy Services Unit should be expanded to include printing electronic articles on a cost-recovery basis.

Having addressed the storage issue and determined how access could work in one scenario, the task force then considered internal processing of electronic journals. We decided to handle them much like serials in other formats. Electronic journals would receive full treatment following CONSER guidelines, including Library of Congress call numbers and subject headings, linking entries, name authority work, etc. The bibliographic record would also describe the means of access in a general note (MARC tag 500) and in a local note (MARC tag 590).

500طbط Mode of access: Electronic mail on BITNET ([userid] @ [node]) and Internet ([userid] @ [node]).

$590 \not b b$ This electronic journal is available for viewing on the University's INFO system on VTVM1 or VTVM2 under the entry INFO LIBRARY E-JOURNL.

Additional VTLS HELP screens would clearly explain what electronic journals are and how to access them. The VTLS MARC holdings record, besides listing the full extent of electronic journal 
holdings available online, would explicitly direct patrons to the electronic journal source text and identify the sites of dedicated access terminals. This information could go into a textual holdings field (MARC tag 866) and would display a public note:

This electronic journal is available for viewing on the University's INFO system on VTVM1 or VTVM2 under the entry INFO LIBRARY E-JOURNL

Terminals are available for free public access in the REFERENCE ROOM and at the SCIENCE REFERENCE DESK

In cooperation with Acquisitions Department personnel, the task force addressed other internal processes such as ordering, receiving, and claiming electronic journals. Here we anticipate making some adjustments for handling serials in this new electronic format.

Orders for new titles would be initiated by subject bibliographers, with the Principal Bibliographer's approval required even for new subscriptions to free journals. When a subscription is placed for a new electronic journal, the ordering clerk would ask the publisher to invoice Business Services (if indeed there is a subscription fee) and to send the text to the VM account maintained by the Acquisitions Department and dedicated to ordering and receiving electronic journals. Serials Receiving staff should open this account weekly to receive the issues - weekly because after one week, unopened files are automatically deleted by the system. These procedures assume a typical case in which publishers distribute journal issues to subscriber mailing lists, with receipt a passive activity for the library once its account has been established.

Somewhat more proactive procedures are required for electronic journals that notify subscribers via e-mail that issues are available, rather than sending issues to each subscriber's user ID. Sometimes an electronic journal issue may be so large that it must be sent in multiple files. In these cases, Serials Receiving will establish procedures for directly accessing the online host accounts and receiving text according to the schedules announced by journal publishers.

In both situations, Serials Receiving staff would give each file of the online issue a cursory check to ensure that basic conventions of text storage had been maintained and that the text is actually eye- 
readable. Serials Receiving staff would record the receipt of a new issue following the procedures now used for serials in all other formats (though electronic journals should be included in our current testing of online check-in). Claiming procedures should be straightforward, calculated by the same means now used for journals in other formats. These checks and procedures will certainly be refined with experience.

New titles would be forwarded to the Serials Cataloging VM account for cataloging and creating the MARC holdings records. Additional issues could be checked-in online and the MARC holdings record updated in Serials Receiving. As usual, Serials Receiving would forward to and notify Serials Cataloging of changes in title, frequency, or numbering. An explanatory e-mail note would 'accompany' the electronic journal when it is forwarded to Serials Cataloging.

The task force has recommended, as an interim measure, that the Database Administration Department be responsible for maintaining the E-JOURNL portion of INFO LIBRARY. At each stage where files are forwarded, the sending unit would purge the text from its own account after receiving e-mail notification from the subsequent unit that text transferred successfully.

In addition to clarifying technical processing, we addressed reference concerns. To handle the new technology the Reference Department has created a separate location, the Electronic Reference Area, with individual workstations that have both dedicated and multiple CD-ROM access. Reference Librarians have also had to become familiar with the new electronic tools, learning a variety of search strategies and commands as well as becoming acquainted with a variety of databases. All of these services require equipment, supplies, and a trained staff, able to train patrons in the use of these information technologies. The task force recommended that the Libraries' administration provide additional resources as the Libraries expand to include newer technologies, so that the introduction of this new service - access to e-journals - does not erode the level of service currently being provided. We included an appendix to our report that lists "resource considerations," because we wanted it to be recognized that monetary as well as organizational resources are necessary to insure the success of this endeavor. 
3. Paul Gherman (University Librarian) to Paul Metz (Principal Bibliographer), 28 September 1990.

4. The task force members included: Bill Dougherty, Beth Hanson (implementation team chair), Buddy Litchfield, Paul Metz (first task force chair), and Kelly Queijo. The implementation team was later joined by Harry Kriz and Bill Kownacki, with input from Eleanor Garrison and Brenda Pratt.

5. "Report of the Task Force on the Electronic Journal," University Libraries, Virginia Polytechnic Institute and State University, 21 April 1991. The report was revised for distribution 17 May 1991.

6. Storage on the university mainframe computer could become a problem if electronic journals become extremely popular and proliferate to such an extent that "old" issues cannot be left online, but must be stored on off-line tapes. These would be loaded for users upon request, but would not be as immediately accessible as current issues. 


\section{A CHANGING WORLD: Proceedings of the NORTH AMERICAN SERIALS INTEREST GROUP, Inc.}

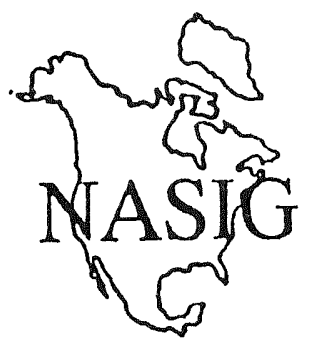

6th Annual Conference

June 14-17, 1991

Trinity University

San Antonio, Texas

Suzanne McMahon

Miriam Palm

Pam Dunn

Guest Editors

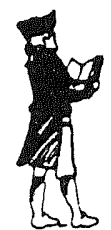

The Haworth Press, Inc. New York 


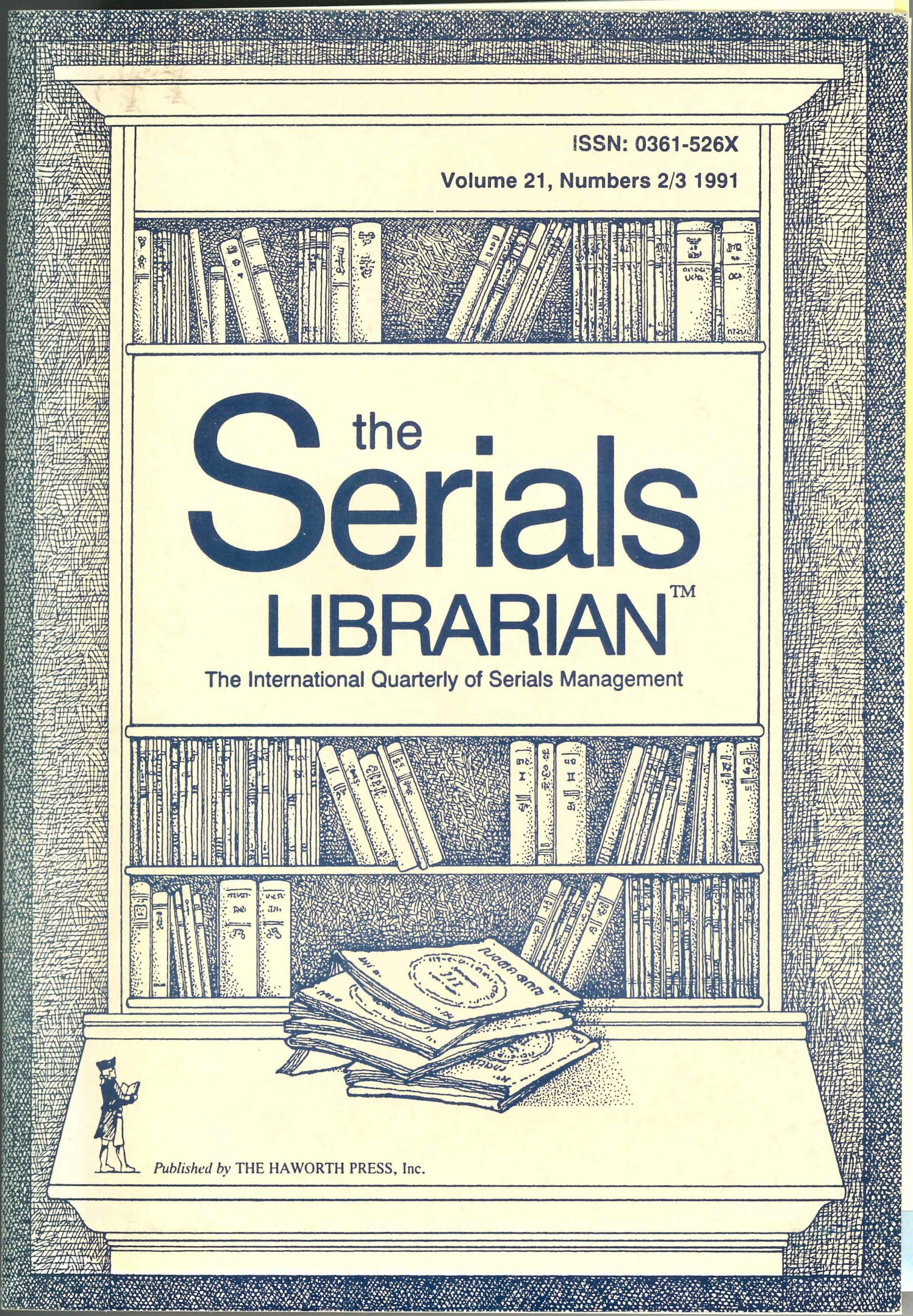

